\title{
THERE ARE KNOTS WHOSE TUNNEL NUMBERS GO DOWN UNDER CONNECTED SUM
}

\author{
KANJI MORIMOTO \\ (Communicated by James E. West)
}

\begin{abstract}
In this paper, we show that there are infinitely many tunnel number two knots $K$ such that the tunnel number of $K \# K^{\prime}$ is equal to two again for any 2-bridge knot $K^{\prime}$.
\end{abstract}

\section{INTRODUCTION}

Let $K$ be a knot in the 3-sphere $S^{3}$, and $t(K)$ the tunnel number of $K$. Here the tunnel number of $K$ is the minimum number of mutually disjoint arcs properly embedded in the exterior of $K$ whose exterior is a handlebody. We call the family of such arcs an unknotting tunnel system for $K$. In particular, we call it an unknotting tunnel for $K$, if the family consists of a single arc. On behavior of the tunnel number of knots under connected sum, the most simple case is:

Theorem 1 ([N], [Sc] and [MS]). Tunnel number one knots are prime.

And in the previous paper, we have shown:

Theorem 2 ([M1]). Let $K_{1}$ and $K_{2}$ be non-trivial knots in $S^{3}$, and suppose $t\left(K_{1} \# K_{2}\right)=2$. Then:

(1) if neither $K_{1}$ nor $K_{2}$ is a 2-bridge knot, then $t\left(K_{1}\right)=t\left(K_{2}\right)=1$ or,

(2) if one of $K_{1}$ and $K_{2}$, say $K_{1}$, is a 2-bridge knot, then $t\left(K_{2}\right) \leq 2$ and $K_{2}$ is prime.

In this paper, we show :

Theorem 3. There are infinitely many tunnel number two knots $K$ such that $t\left(K \# K^{\prime}\right)=2$ for any 2-bridge knot $K^{\prime}$.

\section{KNOT $K_{n}$}

Let $n$ be an integer $(>1)$ and $K_{n}$ the knot illustrated in Figure 1 .

To prove Theorem 3, we show that (1): $t\left(K_{n}\right)=2, \quad(2): t\left(K_{n} \# K\right)=2$ for any 2-bridge knot $K$ and (3) : $K_{n}$ and $K_{n^{\prime}}$ are mutually different types if $n$ and $n^{\prime}$ are mutually different integers $(>1)$.

Received by the editors April 10, 1993.

1991 Mathematics Subject Classification. Primary 57M25.

Key words and phrases. Knots, tunnel numbers, connected sum. 


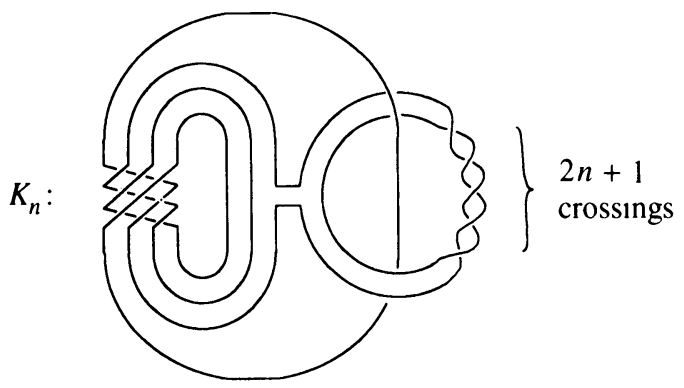

FIGURE 1

Lemma 1. $t\left(K_{n}\right) \leq 2$ and $t\left(K_{n} \# K\right) \leq 2$ for any 2-bridge knot $K$.

Proof. Let $\gamma_{1}$ and $\gamma_{2}$ be the two arcs indicated in Figure 2-(1). Then by the deformation illustrated in Figure 2-(1) through Figure 2-(6), and since the arc $\rho$ indicated in Figure 2-(6) is an unknotting tunnel for the (4, 3)-torus knot (cf. [BRZ]), we see that $c l\left(E\left(K_{n} \# K\right)-N\left(\gamma_{1} \cup \gamma_{2}\right)\right)$ is a genus three handlebody, where $E\left(K_{n} \# K\right)=c l\left(S^{3}-N\left(K_{n} \# K\right)\right)$ and $N(\cdot)$ denotes a regular neighborhood. This shows that $\left\{\gamma_{1}, \gamma_{2}\right\}$ is an unknotting tunnel system for $K_{n} \# K$. Since any 2-bridge knot has such a projection that each block has even crossings, we have $t\left(K_{n} \# K\right) \leq 2$ for any 2-bridge knot $K$. Moreover, since a trivial knot has a 2-bridge decomposition, this inequality implies $t\left(K_{n}\right) \leq 2$. This completes the proof of the lemma.

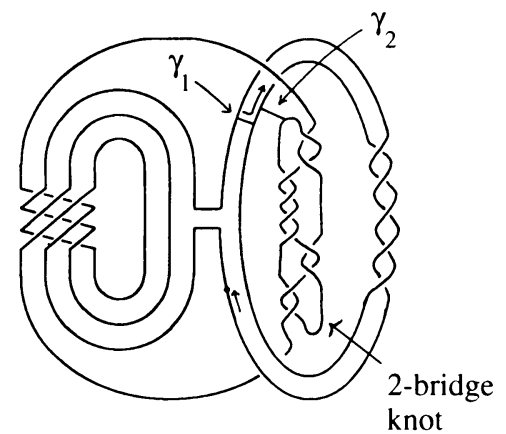

FIGURE 2-(1)

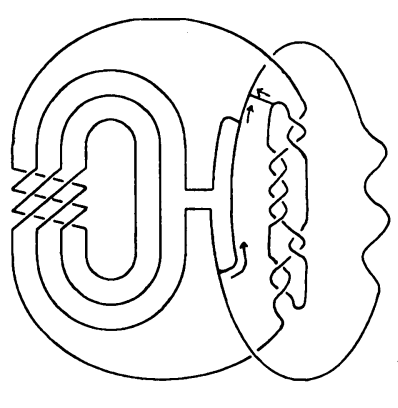

FIGURE 2-(2)

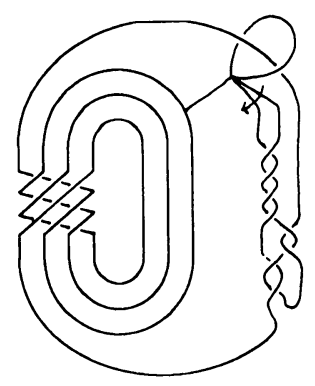

FigURE 2-(4)

FigURE 2-(3) 


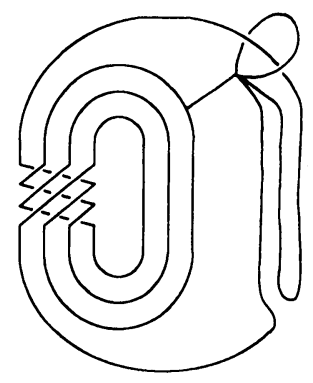

FiGURE 2-(5)

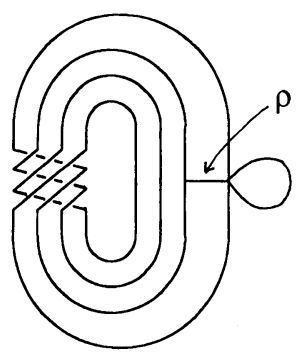

FIGURE 2-(6)

2. TANGLE $T_{m}$

Let $m$ be an integer $(>0)$ and $(B, s \cup t)$, say $T_{m}$, the 2-string tangle illustrated in Figure 3, where $B$ is a 3-ball, $s$ is the trivial arc properly embedded in $B$ and $t$ is the knotted arc properly embedded in $B$. Since there is a disk properly embedded in $B$ which intersects $s \cup t$ in two points and splits $T_{m}$ into two 2-string trivial tangles, we have the next lemma.

Lemma 2. The 2-fold branched covering space of $B$ along $s \cup t$, say $\Sigma_{2}\left(T_{m}\right)$, is a Seifert fibered space over a disk with two exceptional fibers, and the Seifert invariants are $\frac{1}{2}$ and $\frac{-1}{2 m+1}$. Hence if $m \neq m^{\prime}$, then $T_{m}$ is not homeomorphic to $T_{m^{\prime}}$ (cf. [Se]).

Let $S$ be the 2-sphere indicated in Figure 4 (on the next page) and $B_{1}, B_{2}$ the 3-balls bounded by $S$. Put $B_{i} \cap K_{n}=s_{i} \cup t_{i}(i=1,2)$, where $s_{i}$ is a trivial arc and $t_{i}$ is a knotted arc. And let $\alpha_{i}$ be the arc in $\partial B_{i}$ indicated in Figure 5-(1) and in Figure 6-(1) (on the next page) connecting two points in $\partial\left(s_{i} \cup t_{i}\right)$. Then by the deformation illustrated in Figure 5-(1) through 5-(4) and in Figure 6-(1) through 6-(2), we have the next lemma.

Lemma 3. $\left(B_{1}, s_{1} \cup t_{1}\right)$ is homeomorphic to $T_{1},\left(B_{2}, s_{2} \cup t_{2}\right)$ is homeomorphic to $T_{n}$ and $\alpha_{1}$ is identified with $\alpha_{2}$.

Lemma 4. The 2-fold branched covering space of $S^{3}$ along $K_{n}$, say $\Sigma_{2}\left(K_{n}\right)$, is a union of two Seifert fibered spaces $\Sigma_{2}\left(T_{1}\right)$ and $\Sigma_{2}\left(T_{n}\right)$, and is not a Seifert fibered space. Hence the preimage of $S$ is a torus which gives the torus decomposition of $\Sigma_{2}\left(K_{n}\right)$ (cf. [JS] and [Jo]).

Proof. Put $T=\partial \Sigma_{2}\left(T_{1}\right)=\partial \Sigma_{2}\left(T_{2}\right)$. Then $T$ is a separating incompressible torus in $\Sigma_{2}\left(K_{n}\right)$.

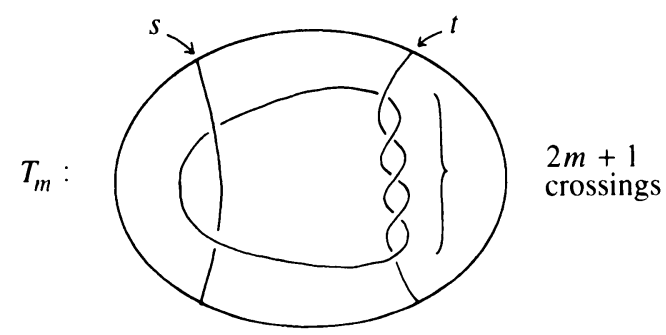

FigURE 3 


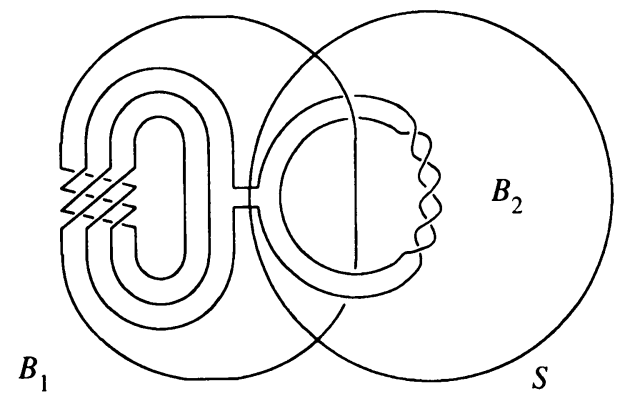

FIGURE 4

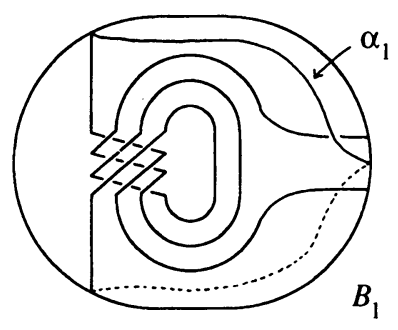

Figure 5-(1)

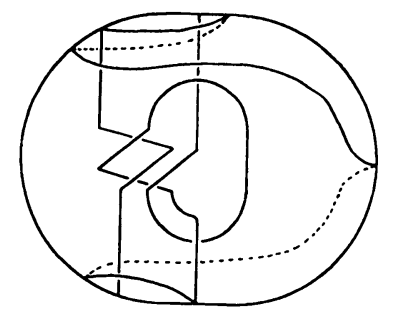

Figure 5-(3)

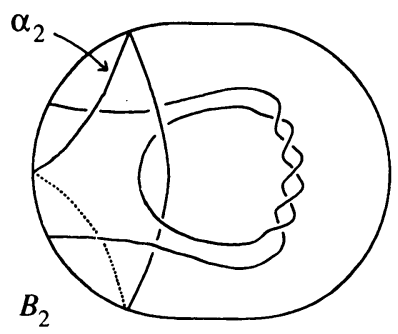

Figure 6-(1)

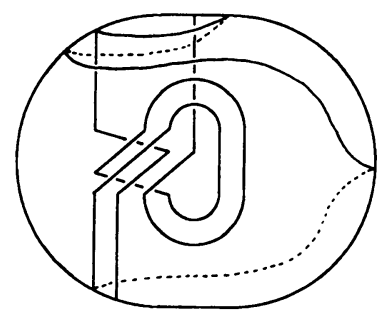

Figure 5-(2)

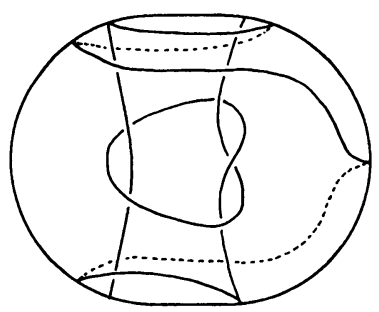

Figure 5-(4)

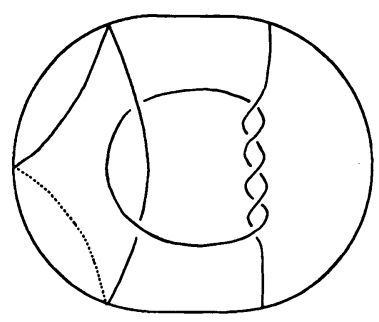

Figure 6-(2)

Suppose $\Sigma_{2}\left(K_{n}\right)$ is a Seifert fibered space. Then by Theorem VI.34 of [Ja], $T$ is saturated in some Seifert fibration of $\Sigma_{2}\left(K_{n}\right)$, or splits $\Sigma_{2}\left(K_{n}\right)$ into two 
twisted I-bundles over a Klein bottle. By Theorem VI.17 of [Ja] and Lemma 2, both $\Sigma_{2}\left(T_{1}\right)$ and $\Sigma_{2}\left(T_{n}\right)$ admit unique Seifert fibrations, and $\Sigma_{2}\left(T_{1}\right)$ is not a twisted I-bundle over a Klein bottle. Hence the Seifert fibration of $\Sigma_{2}\left(T_{1}\right)$ has to extend to the Seifert fibration of $\Sigma_{2}\left(T_{n}\right)$. However, it does not extend because, by the proof of Lemma 3, the preimage of $\alpha_{2}$ is a regular fiber of $\Sigma_{2}\left(T_{n}\right)$, the preimage of $\alpha_{1}$ is not a regular fiber of $\Sigma_{2}\left(T_{1}\right)$ and $\alpha_{1}$ is identified with $\alpha_{2}$. This is a contradiction and completes the proof of the lemma.

The next lemma is an immediate consequence of Birman-Hilden and Viro's result, that is, any genus two orientable closed 3-manifold has an orientation preserving involution which preseves each genus two handlebody setwise (cf. $[\mathrm{BH}]$ and $[\mathrm{V}])$.

Lemma 5. If a knot $K$ has tunnel number one, then there is an orientation preserving involution $h$ of $S^{3}$ such that $h(K)=K$ and the fixed point set of $h$ intersects $K$ in two points.

We note here that the above involution $h$ reverses the orientation of $K$.

\section{Proof of Theorem 3}

Suppose $t\left(K_{n}\right) \leq 1$. Then there is an involution $h$ as in Lemma 5. Let $T$ be the preimage of $S$ in $\Sigma_{2}\left(K_{n}\right)$ and $\tilde{h}$ the lift of $h$, i.e. $\tilde{h}$ is a selfhomeomorphism of $\Sigma_{2}\left(K_{n}\right)$ with $h \circ p=p \circ \tilde{h}$, where $p: \Sigma_{2}\left(K_{n}\right) \rightarrow S^{3}$ is the projection. Then by the uniqueness of torus decomposition, $\tilde{h}$ is isotopic to a self-homeomorphism $\tilde{h}_{*}$ of $\Sigma_{2}\left(K_{n}\right)$ such that $\tilde{h}_{*}(T)=T$. Moreover by [BS], we can choose the isotopy to be equivariant with $p$. Hence we may assume that $h(S)=S$.

Since $n>1$, by Lemma 2 we have $h\left(B_{1}\right)=B_{1}$ and $h\left(B_{2}\right)=B_{2}$. Moreover since $s_{i}$ is a trivial arc and $t_{i}$ is a knotted arc $(i=1,2)$, we have $h\left(s_{1}\right)=$ $s_{1}, h\left(t_{1}\right)=t_{1}, h\left(s_{2}\right)=s_{2}$ and $h\left(t_{2}\right)=t_{2}$. However, since $h$ reverses the orientation of the arcs, each arc has one fixed point. This shows that the fixed point set of $h$ intersects $K_{n}$ in four points. This contradicts Lemma 5 and completes the proof of $t\left(K_{n}\right)=2$. And by Theorem 1, we have $t\left(K_{n} \# K\right)=2$.

Let $n^{\prime}$ be an integer $(>1)$ different from $n$, and let $\left(B_{1}^{\prime}, s_{1}^{\prime} \cup t_{1}^{\prime}\right) \cup\left(B_{2}^{\prime}, s_{2}^{\prime} \cup t_{2}^{\prime}\right)$ be the tangle decomposition of $\left(S^{3}, K_{n^{\prime}}\right)$ corresponding to that of $\left(S^{3}, K_{n}\right)$. Suppose there is a homeomorphism $f$ of $\left(S^{3}, K_{n}\right)$ to $\left(S^{3}, K_{n^{\prime}}\right)$. Then by the same argument as above, we may assume that $f\left(B_{1}\right)=B_{1}^{\prime}$ and $f\left(B_{2}\right)=B_{2}^{\prime}$. Hence $T_{n}$ is homeomorphic to $T_{n^{\prime}}$. This contradicts Lemma 2 and completes the proof of Theorem 3.

Remark. (1) Since the tangle $T_{m}$ is prime, the fact " $t\left(K_{n}\right)=2$ " is an immediate consequence of Theorem 2.3 of [Sc], that is " Tunnel number one knots are doubly prime". In this paper, we showed that $K_{n}$ is not strongly invertible.

(2) Let $K$ be a tunnel number two knot. In the forthcoming paper [M2], we show that if $t\left(K \# K^{\prime}\right)=2$ for some 2-bridge knot $K^{\prime}$, then $t\left(K \# K^{\prime}\right)=2$ for any 2-bridge knot $K^{\prime}$.

\section{ACKNOWLEDGEMENT}

This work was carried out while I was visiting the University of Texas at Austin. I would like to express my gratitude to the University and to Professor Cameron Gordon for their hospitality and encouragement. 


\section{REFERENCES}

[BH] J. S. Birman and H. M. Hilden, Heegaard splittings of branched covering space of $S^{3}$, Trans. Amer. Math. Soc. 213 (1975), 315-352.

[BRZ] M. Boileau, M. Rost, and H. Zieschang, On Heegaard decompositions of torus knot exteriors and related Seifert fibre spaces, Math. Ann. 279 (1988), 553-581.

[BS] F. Bonahon and L. Seibemann, Geometric splittings of knots, and Conway's algebraic knots, preprint.

[Ja] W. Jaco, Lectures on three manifold topology, CBMS Regional Conf. Ser. in Math., vol. 43, Amer. Math. Soc., Providence, RI, 1980.

[JS] W. Jaco and P. B. Shalen, Seifert fibered spaces in 3-manifolds, Mem. Amer. Math. Soc., No. 220 (1979).

[Jo] K. Johannson, Homotopy equivalence of 3-manifolds with boundaries, Lecture Notes in Math., vol. 761, Springer-Verlag, Berlin and New York, 1979.

[M1] K. Morimoto, On the additivity of tunnel number of knots, Topology Appl. 53 (1993), 37-66.

[M2] Characterization of tunnel number two knots which have the property " $2+1=2$ ", Topology Appl. (to appear).

[MS] K. Morimoto and M. Sakuma, On unknotting tunnels for knots, Math. Ann. 289 (1991), 143-167.

[N] F. Norwood, Every two generators knot is prime, Proc. Amer. Math. Soc. 86 (1982), 143-147.

[Sc] M. Scharlemann, Tunnel number one knots satisfy the Poenaru conjecture, Topology Appl. 18 (1984), 235-258.

[Se] H. Seifert, Topologie dreidimensionaler gefaserter Raume, Acta. Math. 60 (1933), 147-238.

[V] O. J. Viro, Linkings, two sheeted branched coverings and braids, Mat. Sb. 87 (1972), 216-228.

Department of Mathematics, Takushoku University, Tatemachi, Hachioj, Tokyo 193, JAPAN

E-mail address: morimoto@la.takushoku-u.ac.jp 